\title{
Combined selective plane illumination microscopy and FRAP maps intranuclear diffusion of NLS-GFP
}

Chad Hobson, E. Timothy O'Brien III, Michael Falvo, Richard Superfine The University of North Carolina at Chapel Hill, USA

\section{Abstract Text}

Since its initial development in 1976, fluorescence recovery after photobleaching (FRAP) has been one of the most popular tools for studying diffusion and protein dynamics in living cells. Its popularity is derived from the widespread availability of confocal microscopes and the relative ease of the experiment and analysis. FRAP, however, is limited in its ability to resolve spatial heterogeneity. Here, we combine selective plane illumination microscopy (SPIM) and FRAP to create SPIM-FRAP [1], wherein we use a sheet of light to bleach a 2D plane and subsequently image the recovery of the same image plane. This provides simultaneous quantification of diffusion or protein recovery for every pixel in a given 2D slice, thus moving FRAP measurements beyond these previous limitations. To perform these measurements, we use our custom, open-top, single-objective SPIM system [2]. We demonstrate this technique by mapping both intranuclear diffusion of NLS-GFP and recovery of 53BP1-mCherry, a marker for DNA damage, in live MDA-MB-231 cells. SPIM-FRAP proves to be an order of magnitude faster than fluorescence correlation spectroscopy (FCS) based techniques for such measurements. We observe large length-scale ( $>\sim 500 \mathrm{~nm}$ ) heterogeneity in the recovery times of NLS-GFP, which is validated against simulated data sets. 2D maps of NLS-GFP recovery times showed no pixel-by-pixel correlation with histone density. Although, slower diffusion was observed in nucleoli. Additionally, recovery of 53BP1-mCherry was observed to be slowed at sites of DNA damage. We finally developed a diffusion simulation for our SPIM-FRAP experiments to compare across techniques. Our measured diffusion coefficients are on the order of previously reported results, thus validating the quantitative accuracy of SPIM-FRAP relative to well-established methods. With the 
recent rise of accessibility of SPIM systems, SPIM-FRAP is set to provide a straightforward means of quantifying the spatial distribution of protein recovery or diffusion in living cells. 